\title{
The wisdom of patients and families: ignore it at our peril
}

\section{Liam J Donaldson}

\section{Correspondence to}

Professor Liam Donaldson, Department of Surgery and Cancer, Imperial College London, St Mary's Campus, London W2 1NY, UK; I. donaldson@imperial.ac.uk

Accepted 8 July 2015 Published Online First 22 July 2015

\section{SLinked}

http://dx.doi.org/10.1136/ bmjqs-2015-003980

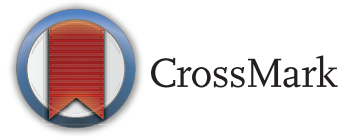

To cite: Donaldson LJ. BMJ Qual Saf 2015;24:603-604.

\section{PATIENT-REPORTED INCIDENTS HELP TO UNDERSTAND AND REDUCE HARM}

Health system leaders, and those managing healthcare organisations, are increasingly trying to find the right way to use the views and experience of patients to make the services that they provide better and safer. The traditional path is to start with data. But the days when a provider of care could pride itself as being patient-centred purely by capturing patient feedback on its services have long gone. Today, the emphasis is on outcomes defined by patients: so-called patient-reported outcome measures. ${ }^{1}$ For example, The International Consortium for Health Outcomes Measurement ${ }^{2}$ defines outcome as: 'The results people care about most when seeking treatment, including functional impairment and the ability to live normal, productive lives'.

There can be no area that people receiving health services should care more deeply about than being protected from the risk of avoidable harm. For the past decade, governments, health systems, providers of care and professional bodies around the world have placed a great deal of faith in incident reporting systems as the main route to safer care. Large volumes of such reports have been accumulated: in England and Wales, for instance, the database of patient safety incidents stands at 12 million. ${ }^{3}$ Yet, taking a global perspective, there are too few examples of where a sustained reduction in risk can be unambiguously attributed to the fruitful analysis of incident data. Some question the purpose of continuing to invest time and money in this endeavour, while others believe that the potential can still be realised. ${ }^{4}$

Frontline healthcare professionals submit the majority of all incident reports; the narrative elements describing the failure of care are based on their insights. Understandably, such accounts are generally factual, clinical and technical. Feelings and emotions play little part in conveying what can be dramatic and life-changing events for patients and families. This latter aspect of harm tends to be captured in what the patient safety world terms 'patient stories', first-hand accounts by those who have been the victims, communicated in papers, in books and at conferences, ${ }^{5}$ but which are separate from the routine, daily flow of incident reports.

A study based on patient-reported adverse event data, ${ }^{6}$ gathered on a voluntary basis, provides a comparison with research reports based on traditional patient safety incident data. $^{7}$ There are limitations: under-reporting, selectivity and lack of a reliable denominator-but these are also present in many studies based on reporting by clinical staff. What is remarkable about the analysis of these patient-initiated reports is that it shows broad consistency with the major categories of harm captured in established patient safety reporting systems (eg, healthcare-associated infection, medication error, misdiagnosis, wrong-site surgery) while also capturing the psychological, social and economic impact. The relatively small sample size and limited geographical coverage mean that caution is necessary in claiming universality of the messages, but the findings will ring many bells with those in the field of patient safety.

For those of us who have listened with deep concern to many individual accounts by patients and family members in conference presentations or during private conversations, it is particularly chilling to see how the aggregated experience of this study is so similar to the poignant individual accounts. It confirms the very strong impression that too many healthcare organisations espouse the goal of safer care while regarding harm as the cost of doing business. A failure of providers to respond appropriately to the suffering that they have caused, a sense of 
abandonment, disrespect, a failure to listen, secrecy, long-term psychological and financial consequences were all key elements of the patients' reports in the study. There was also a strongly expressed desire for openness and transparency, a long-standing aspect of the culture of healthcare worldwide that is too often lacking and that has led to calls for a statutory duty of candour $^{8}$ or similar disclosure processes.

The study also analysed the proposals for preventing harm contained in the patient reports. Again these were very consistent with professionally defined system design solutions (eg, the placement of containers for alcohol rubs).

Though some might be surprised that patients' reports of unsafe care can contribute to identifying types of harm, to helping understand causation and to identifying solutions, few could doubt the authenticity of the damage it causes in human terms. Yet, too often, this is seen as something to be 'handled' with a degree of wariness, or even worse, simply to be acknowledged with ritual expressions of regret rather than seeing it as part of the overall process of learning. Indeed, it is striking how a catastrophic event such as an avoidable death within a healthcare organisation infrequently leads to transformational change in the approach to safety; the re-orientation of services as a result of the deaths of Betsy Lehman ${ }^{9}$ and Josie King ${ }^{10}$ appear to be among the small number of exceptions. Harm almost always comes in more than one wave. The first is physical, the second emotional and psychological and the third, and later waves, produce the long-term trauma that haunts all who are part of a tragic event. Too often, healthcare organisations, through their inept and unfeeling responses, amplify these later waves of harm rather than mitigating their damaging effects.

A patient safety incident reporting and analysis process should fulfil five main purposes ${ }^{11}$ : a public accountability function, a response to the patients and families involved, a communications alert route, a barometer of risk within the healthcare system and a foundation for learning and improvement. Engagement with patients and families, particularly those with experience of harm, enriches the approach in all of these areas. Incident reporting systems that do not include and promote reports from patients in future should be regarded as not fit for purpose.

Learning from patients' and families' experience of harm can help to limit the scale of that harm for future patients, it can identify the need for practical action to reduce future risk, but most importantly it can motivate and inspire all staff to continue the journey to safer healthcare.

Competing interests None declared.

Provenance and peer review Commissioned; internally peer reviewed.

\section{REFERENCES}

1 Nelson EC, Effimovaka E, Lind C, et al. Patient reported outcome measures in practice. BMJ 2015;350:g7818.

2 International Consortium for Health Outcomes Measurement. http://www.ichom.org/why-we-do-it/ (accessed 6 Jul 2015).

3 NHS England. National reporting and learning system. 2015. http://www.nrls.npsa.nhs.uk/report-a-patient-safety-incident/ about-reporting-patient-safety-incidents/ (accessed 6 Jul 2015).

4 Donaldson LJ, Panesar SS, Darzi A. Patient-safety-related hospital deaths in England: thematic analysis of incidents reported to a national database, 2010-2012. PLoS Med 2014;11: e1001667.

5 McIver S, Wyndham R. After the error; speaking out about patient safety to save lives. Toronto: ECW Press, 2013.

6 Southwick FS, Cranley NM, Hallisy JA. A patient-initiated voluntary online survey of adverse medical events: the perspective of 696 injured patients and families. BMJ Qual Saf $2015 ; 24: 620-9$.

7 Cousins DH, Gerret D, Warner B. A review of medication incidents reported to the National Reporting and Learning System in England and Wales over six years (2005-2010). Br J Clin Pharmacol 2012;74:597-604.

8 Health and Social Care Act 2008 (Regulated Activities) Regulations 2014: Regulation 20 Duty of candour.

9 Conway JB, Weingart S. Organizational Change in the Face of Highly Public Errors I. The Dana-Farber Cancer Institute Experience. Morbidity and mortality rounds on the web. Washington DC: AHRQ, 2005.

10 Ayd MA. A remedy of errors. Johns Hopkins Medicine. Baltimore: Johns Hopkins University, 2004.

11 Donaldson LJ. The right time, the right place: an expert examination of the application of health and social care governance arrangements for ensuring quality of care provision in Northern Ireland. Belfast: Department of Health, Social Services and Public Safety, 2014. 